\title{
Régime alimentaire d'un ver de terre anécique des savanes colombiennes - une remise en question des types écologiques
}

\author{
Lucero Mariani $^{1^{*}}$, Nicolas Bernier ${ }^{2}$, Juan José Jiménez ${ }^{3}$, Thibaud Decaëns ${ }^{4}$ \\ 1 Laboratoire d'Ecologie des Sols Tropicaux, IRD, 32 Av. H. Varagnat, 93143 Bondy Cedex, \\ France. \\ *Correspondance et tirés à part : lucero.mariani@bondy.ird.fr, \\ téléphone : 33 (0)1 480259 60, télécopie : 33 (0) 148473088 \\ 2 Laboratoire d'Ecologie Générale, Muséum National d'Histoire Naturelle, 4 Av. du Petit \\ Château, 91800 Brunoy, France. \\ 3 Soil and Plant Nutrition Unit, CIAT, AA 6713 Cali, Colombia. \\ 4 Laboratoire d'Ecologie, Université de Rouen, F-76821 Mont Saint Aignan Cedex, France \\ Running title : tropical anecic earthworms diet and ecological groups
}

Résumé - La classification écologique des vers de terre basée sur des caractères morphologiques permet de décrire leur fonction dans le sol. Cependant ces relations ont rarement été validées. Les gésiers du ver de terre Martiodrilus carimaguensis ont été étudiés pour vérifier que sa morphologie d'anécique l'amenait bien à consommer de la litière mélangée au sol superficiel.

Les pourcentages volumiques des fragments végétaux, des racines, de la matière organique amorphe et des minéraux du contenu du gésier de 13 individus adultes, déterminé par l'observation directe à la loupe et au microscope, sont respectivement de $63,5,6,5$ et $30 \% \mathrm{~V} / \mathrm{V}$. Quatre-vingt pourcents des fragments végétaux sont microscopiques et incorporés au sein de déjections de vers de terre. La coprophagie et la rhizophagie sont les comportements alimentaires dominants de $\underline{M}$. carimaguensis. Toutefois ses turricules attestent d'une variabilité de son alimentation qui pourrait résulter de l'adoption transitoire d'un régime d'anécique typique.

Mots clés : types écologiques / groupes fonctionnels / vers de terre anéciques / régimes alimentaires / contenus intestinaux / coprophagie / sols tropicaux / savanes

\begin{abstract}
Diet of an anecic earthworm from the Colombian savannas : questioning ecological groups. An ecological classification based on a set of morphological characters may be used to describe earthworm functions in soil. However these relationships have been seldom verified. Gizzards of the earthworm Martiodrilus carimaguensis (Glossoscolecidae) were studied to find out if its anecic morphology was in agreement with its diet (shallow litter remains and deep organo-mineral material).

By direct observation of 13 adults' gizzard contents with a dissecting and a phase contrast microscope, the volumetric percentage of plant fragments, roots, amorphous organic matter and mineral particles reached respectively 63, 5, 6.5 and $30 \% \mathrm{~V} / \mathrm{V}$. Eighty percent of plant fragments were microscopic, and mostly incorporated into the organo-mineral earthworm casts well preserved in the gizzard. Coprophagy and rhizophagy were the dominant feature of $\underline{M}$. carimaguensis diet. Yet, the casts produced revelaed a high variability in its diet, what could be related to transitional adoption of a typical anecic diet during some periods.
\end{abstract}


Key words : ecological groups / anecics earthworms / gut contents / diet / coprophagy / tropical soils/ savanna

\section{Abridged version :}

Endogeic and anecic earthworms are ecosystem engineers that produce remaining macropores and organo-mineral structures that can influence soil hydraulic properties, soil organic matter dynamic, and, in fine, plant growth and soil conservation. However, high input monocropping leads to a reduction of their populations to densities at which their impact is not significant. Indirect management of their activities through agricultural practices seems to be the most appropriate way to sustain faunal activity. Knowledge of key species' feeding habits and food requirements is needed to evaluate and improve the carrying capacity of agricultural fields for them. This will also help evaluating the amount of surface litter that is actually incorporate into soils.

At the study site in Colombia, where different crop-livestock systems are used, Martiodrilus carimaguensis (Glossoscolecidae) seems to be a good candidate for indirect management in order to improve sustainable use of the soil. This species constitutes a large proportion of the biomass of soil macroinvertebrate in intensive pastures and seems to be able to colonise adjacent intensive cultures. It produces large casts at the soil surface that are sources of mineral nitrogen and organic carbon when fresh, i.e., during one or two weeks after deposition. When casts dry, carbon is protected from decomposition inside them. M. carimaguensis is a large-sized species, $9 \mathrm{~mm}$ in diameter, $190 \mathrm{~mm}$ long and a fresh weight of $11 \mathrm{~g}$ when fully adult on average. It has been classified as anecic according to morphological traits (dorsal dark colour with sharp anteroposterior gradients, body end flattened) habitat characteristics (subvertical and semi-permanent burrow) and adaptive strategies (K strategy and true diapause during the dry season). The diet of this species is studied in order to evaluate the quality of their food requirements and verify that it are truly belongs to the anecic group as suggested by its external characters.

Earthworms were collected by the formalin method during the rainy season. Gizzard contents of 13 adults ( 8 from an introduced pasture and 5 from a native savanna) were examined under a dissecting microscope (magnification $\mathrm{x} 40$ ) and a phase contrast microscope (magnification $\mathrm{x} 630$ ) for organo-mineral matter smaller than $500 \mu \mathrm{m}$ which is reported as aggregates in dissecting microscope observations. The volumetric percentage of 12 components was measured using the counting point method with about 100 counts per sample. Particle size was also registered in 11 classes ranging from $0.8 \mu \mathrm{m}$ to $3 \mathrm{~mm}$ for all components but amorphous ones.

Individual variability was noticeably high particularly for macroscopic plant fragments which ranged from 0 to $48 \% \mathrm{~V} / \mathrm{V}$. However, aggregates were more abundant (median $=77 \% \mathrm{~V} / \mathrm{V}$ ) than macroscopic plant fragments and minerals (14 and $11 \% \mathrm{~V} / \mathrm{V}$ respectively). Macroscopic plant fragments mostly comprised roots except for one individual from the introduced pasture. All individuals ingested casts of smaller earthworms and microscopic plant fragments were abundant $(48 \% \mathrm{~V} / \mathrm{V})$. Is not for the same individual that no rhizophagy and no coprophagy were observed. As we had observed under the phase contrast microscope, organic matter was better mixed with soil mineral phase for individuals of the native savanna : their gizzards contained more organomineral complexes (18\% versus $6 \%$ ), more plant fragments $(8 \%$ versus $2 \%$ ) and minerals forming complex compounds ( $64 \%$ versus $2 \%$ for native savanna and improved pasture respectively). Earthworms from the native savanna had also less litter, ca. $14 \mathrm{~g} \mathrm{~m}^{-2}$, than those from improved pasture, ca. $97 \mathrm{gm}^{-2}$. 
The volumetric percentages obtained in our study for plant microscopic debris, well-decomposed organic matter, mineral matter and macroscopic roots was equal to $38.5,7,54$ and $3 \%$ in weight, respectively. Most microscopic plant debris were incorporated into intact organo-mineral faecal pellets before ingestion. The abundance of particles in gizzard contents displayed a bimodal curve with two privileged size of particles, $0.8 \mu \mathrm{m}$ and $0.08 \mathrm{~mm}$ to $0.475 \mathrm{~mm}$. The two scales of observation probably correspond to 2 sources of food for $\mathrm{M}$. carimaguensis, casts and roots for organic matter and casts and coarse feed sand for mineral particles.

The amount of surface litter directly ingested by $\underline{\mathrm{M}}$. carimaguensis is likely around $3 \% \mathrm{~g} / \mathrm{g}$ of its gizzard content. Only one individual from the introduced pasture had an anecic diet i. e. a gizzard content dominated with aerial plant fragments.

Gizzard contents represent the food ingested in a few minutes whereas casts integrate diet characteristics over several days. Unpublished analysis of $\underline{M}$. carimaguensis casts proved an enrichment of seeds content compared with bulk soil that our study was not able to confirm. Thin sections in casts showed an accumulation of small plant fragments veru unevenly distributed. $\underline{\mathrm{M}}$. carimaguensis diet may vary during the nycthemeral period being either a true anecic one (with litter ingestion) or a coprophagic one (probably with a selective ingestion of casts at the soil surface). All the earthworms studied here were collected around 8-10 a.m., thus the large variability in gizzard content of our data is not of nycthemeral origin.

\section{Introduction}

Les vers de terre exercent d'importantes régulations sur les propriétés physiques, la dynamique de la matière organique et la croissance des plantes et constituent de ce fait une ressource potentielle de grand intérêt pour la durabilité de l'agriculture [1]. Des techniques d'inoculation de vers de terre au champ se sont avérées rentables malgré leur coût élevé pour des plantations à forte valeur ajoutée [2]. Pour les cultures les plus répandues telles que les céréales, ces techniques ne sont pas applicables et la solution passe probablement par une gestion indirecte des populations de macroinvertébrés du sol via l'aménagement des pratiques agronomiques. Une bonne connaissance des habitudes alimentaires des espèces cibles est indispensable pour connaître et éventuellement augmenter la capacité de charge des parcelles cultivées pour la faune lombricienne.

Le régime alimentaire est un élément essentiel des classifications fonctionnelles. Bouché [3] a proposé une typologie reliant certains caractères morphologiques des lombrics à leur régime alimentaire et, dans une certaine mesure, à leur fonction supposée dans le sol. Les épigés, reconnaissables à leur pigmentation homogène, vivent dans la litière ou dans le sol immédiatement sous-jacent et consomment des débris végétaux. Les endogés, vers non pigmentés à musculature peu développée, consomment essentiellement du sol et des racines. Les anéciques ont une pigmentation plus marquée dorsalement et dans la partie antérieure, et présentent une musculature fortement développée en rapport avec leurs activités d'enfouissement de litière dans le sol. Les données quantitatives précisant le régime alimentaire des vers de terre anéciques ne concernent pas plus d'une demi-douzaine d'espèces dont une seule tropicale [4-12]. Dans l'optique d'une gestion écologique des agrosystèmes il est essentiel de savoir dans quelle mesure les critères morphologiques définis par Bouché [3] sont de bons indicateurs de leur régime 
alimentaire, et de préciser l'origine et le devenir de cette matière organique une fois effectué le transit intestinal.

Le ver de terre anécique Martiodrilus carimaguensis (Glossoscolecidae) est une espèce native des savanes de l'est de la Colombie. Il s'agit d'un ver de grande taille, $9 \mathrm{~mm}$ de diamètre, $190 \mathrm{~mm}$ de long et $11 \mathrm{~g}$ à l'état adulte [13]. Cette espèce a été choisie pour cette étude car elle présente des biomasses très importantes dans les pâturages intensifs et colonise régulièrement les cultures intensives adjacentes [13,14]. Ce ver produit des galeries sub-verticales ouvertes à la surface et surmontées de gros turricules qui peuvent résister plusieurs mois à la désagrégation [15]. Ces turricules sont une source de nutriments assimilables quand ils sont frais puis une réserve de matière organique de bonne qualité une fois secs [16]. Diverses observations ont montré un effet positif des turricules sur la biomasse racinaire ainsi que sur la densité de certains groupes de macroinvertébrés épigés et anéciques [17]. A l'échelle de petites surfaces expérimentales au

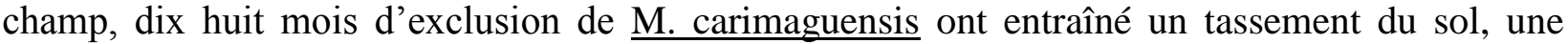
diminution de la teneur en carbone, une augmentation de la saturation aluminique du complexe absorbant, une diminution de la biomasse herbacée et l'augmentation de la proportion d'espèces adventices dans la végétation [18].

\section{Matériels et méthodes}

\subsection{Site d'étude}

Cette étude a été réalisée au centre de recherche de Carimagua (CORPOICA-CIAT) dans les Llanos Orientales de Colombie $\left(4^{\circ} 37^{\prime} \mathrm{N}, 71^{\circ} 19^{\prime} \mathrm{O}\right.$ et $175 \mathrm{~m}$ d'altitude) avec des savanes non inondables et entretenues par des feux comme végétation spontanée dominante. La pluviométrie annuelle et la température moyenne sont respectivement de $2280 \mathrm{~mm}$ et $26^{\circ} \mathrm{C}$ (données non publiées du CIAT). Une saison sèche bien marquée survient de décembre à mars. Les sols sont des oxisols caractérisés par une structure bien agrégée et une très faible fertilité chimique, une forte acidité $\left(\mathrm{pH}\left(\mathrm{H}_{2} \mathrm{O}\right)\right.$ autour de 4,5), une toxicité aluminique élevée (plus de $90 \%$ du complexe absorbant du sol saturé par $\mathrm{Al}^{3+}$ ) et une très faible teneur en cations échangeables $(\mathrm{Ca}, \mathrm{Mg}$ et $\mathrm{K}$ ) (données non publiées du CIAT).

Les parcelles échantillonnées font partie d'un essai agro-pastoral multidisciplinaire visant à étudier l'effet de l'intensification agricole sur le fonctionnement du sol dans les savanes Sud Américaines. Plusieurs agrosystèmes sont testés parmi lesquels des cultures annuelles intensives, des pâturages intensifs et des savanes naturelles comme témoins.

Les deux sites choisis dans cette étude sont 1) une savane naturelle non pâturée mais brûlée annuellement pendant la saison sèche, où dominent les graminées Trachypogon vestitus Anderss,

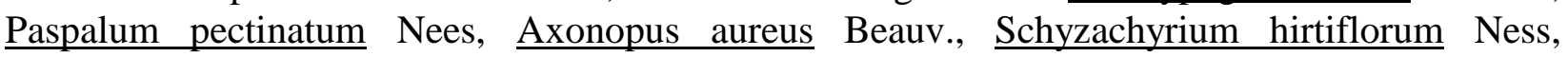
Gymnopogon foliosus Nees et Hiptis conferta Pohl ex Benth. 2) un pâturage semé en 1993 et protégé du feu, associant la graminée allochtone Brachiaria humidicola Rendle avec les légumineuses Arachis pintoi Krap \& Greg, Desmodium ovalifolium Guill. \& Perr. et Stylosanthes capitata Vog. Dans le pâturage amélioré la végétation forme une couverture fermée avec des racines profondes alors que les touffes restent éparses et les racines superficielles en savane spontanée [19]. Le sol est plus riche en carbone dans le pâturage amélioré, 3,7\% contre 2,83\% 
dans la savane, pour les 5 premiers centimètres [16]. La capacité de soutien vis à vis des bovins en saison humide est de 2 unités animales à l'hectare en pâturage amélioré, contre 1 pour 2 ou 3 hectares en savane naturelle ( 1 unité animale $=250 \mathrm{~kg}$ ). La capacité de soutien pour les vers de terre est quatre fois plus élevée en pâturage amélioré (respectivement 1.6 et 0.4 individus au $\mathrm{m}^{2}$ [13] correspondant à 24 et $6 \mathrm{~g}$ au $\mathrm{m}^{2}$ ).

\subsection{Echantillonnage des vers de terre, de la matière organique légère du sol et de la litière}

Durant le mois d'août 1999, des adultes sont collectés dans chaque parcelle en versant une solution de formol diluée $(0,2 \%)$ dans les galeries. Les individus extraits sont immergés dans une solution à proportions égales de formol $4 \%$ et d'alcool $70 \%$ puis conservés dans l'alcool à $70 \%$. Ils sont ensuite pesés et mesurés. Les captures ont toutes été réalisées en début de matinée, sur une période totale n'excédant pas deux semaines.

La litière présente sur le sol est collectée en utilisant deux quadrats circulaires en métal disposés concentriquement autour de l'orifice de chaque galerie de ver de terre, respectivement à $25 \mathrm{~cm}$ et $35 \mathrm{~cm}$ de l'orifice. La première surface est un disque de $50 \mathrm{~cm}$ de diamètre et d'approximativement $0.193 \mathrm{~m}^{2}$ en fonction du diamètre du turricule, la deuxième est une couronne de $0,189 \mathrm{~m}^{2}$. Nous considérons que la quantité de litière collectée dans le cercle intérieur est caractéristique de la galerie (litière galerie) tandis que celle correspondant à la couronne extérieure est caractéristique de la parcelle (litière témoin). La litière est lavée, séchée au four à $75^{\circ} \mathrm{C}$ pendant 2 jours puis pesée.

Un cylindre de sol témoin de $15 \mathrm{~cm}$ de hauteur et $10 \mathrm{~cm}$ de diamètre est prélevé à $5 \mathrm{~m}$ de chaque galerie échantillonnée. La matière organique légère du sol supérieure à $0,125 \mathrm{~mm}$ est isolée par lavage et tamisage en 4 classes de taille, $>2 \mathrm{~mm}, 1-2 \mathrm{~mm}, 0,5-1 \mathrm{~mm}$ et $0,125-0,5 \mathrm{~mm}$. Chaque fraction est séchée au four à $75^{\circ} \mathrm{C}$ pendant 2 jours puis pesée.

Nous avons également constitué une collection de référence des espèces végétales les plus abondantes dans les 2 parcelles étudiées en conservant dans l'alcool à $70 \%$ leurs parties aériennes et souterraines.

\subsection{Contenus des gésiers}

Nous avons isolé le contenu du gésier de 8 adultes du pâturage amélioré et de 5 adultes de la savane naturelle, ce déséquilibre de l'échantillonnage étant dû à des contraintes techniques (des gésiers vides et des détériorations lors de la manipulation). Les contenus sont observés à la loupe binoculaire $(\mathrm{x} 40)$ pour les composants supérieurs à $50 \mu \mathrm{m}$ puis au microscope à contraste de phase (x630) pour les éléments de plus petite taille [12]. Le gésier contient l'ingestat le moins transformé [7].

L'observation à la loupe a permis de séparer les fragments végétaux, les minéraux (contenant du fer ou non), les agrégats organo-minéraux (masse organo-minérale de texture fine aux éléments non identifiables), le charbon et les déjections holorganiques. Les fragments végétaux macroscopiques ont été classés suivant leur origine (racine, parties aériennes et indéterminée). La fixation du matériel dans l'éthanol à $95 \%$ a permis l'observation des structures organo-minérales et leur classification en agrégats galbésprovenant de déjections de vers de terre plus petits, en agrégats anguleux, et en agrégats non classables (probablement endommagés par la dissection et 
la conservation). Au microscope, ont été identifiés des fragments végétaux libres, du charbon libre, des minéraux libres, de la matière organique amorphe isolée et, incorporés au seins de complexes organo-minéraux, des fragments végétaux, des minéraux et de la matière organique amorphe [12]. Le pourcentage volumique de chaque catégorie a été estimé par la méthode de comptage par points à l'aide d'une grille transparente. Le point échantillonné est choisi au hasard car la mise au point est d'abord faite sur la grille puis sur la préparation. Dans les deux cas, l'ensemble de la préparation est parcouru avec environ 100 points par comptage. Cette méthode comprend un risque de surestimation des éléments allongés ou aplatis que nous supposeront jugulé par leur disposition relativement aléatoire dans la préparation. La taille des éléments figurés et des agrégats galbés est notée selon 12 classes allant de $0,8 \mu \mathrm{m}$ à $0,08 \mathrm{~mm}$ pour le microscope et de $0,025 \mathrm{~mm}$ à $3 \mathrm{~mm}$ pour la loupe. Ces deux fenêtres d'observation se superposent partiellement, les classes de tailles $0,025 \mathrm{~mm}$ à $0,05 \mathrm{~mm}$ et $0,05 \mathrm{~mm}$ à $1 \mathrm{~mm}$ étant communes. Les tests de comparaison conduisent à choisir les observations au microscope pour la classe 0,025 - 0,05 mm, la loupe conduisant à une sous-estimation importante, tandis que pour la classe supérieure le choix des observations à la loupe est arbitraire les deux mesures n'étant pas statistiquement différentes.

Les observations à la loupe se font sur l'ensemble du contenu du gésier (moins d'un $\mathrm{cm}^{3}$ ) et celles au microscope sur un aliquote organo-minéral prélevé au hasard sur plusieurs agrégats. Les pourcentages calculés au microscope sont corrigés au prorata des valeurs des agrégats sauf pour les comparer aux observations faites à la loupe.

La provenance anatomique (parties aériennes ou souterraines) des fragments végétaux observés à la loupe a été identifiée à l'aide de la collection de référence.

\subsection{Analyses statistiques}

Les données ont été transformées par la procédure de Box et Cox afin de réduire l'asymétrie et l'aplatissement des distributions [20], à l'aide du progiciel $\mathrm{R}$ [21]. Cependant la faible taille des échantillons et le non-respect de l'hypothèse d'homogénéité des variances pour certaines variables nous ont conduit à appliquer des tests non-paramétriques [22]. Le test U de WilcoxonMann-Whitney a été utilisé pour comparer les échantillons des 2 parcelles et les classes de taille répétées-communes_entre le microscope et la loupe. Des tests de Wilcoxon pour échantillons appariés ont été utilisés pour comparer certaines variables (les pourcentages de racines et de parties aériennes). Des corrélations non-paramétriques (de rang) entre les quantités de litière, les masses de matière organique légère du sol, les masses des individus et les caractéristiques des gésiers ont été utilisées pour expliquer la variabilité inter- et intra- groupe.

Ces tests non-paramétriques sont effectués sur les variables ou les groupes 2 à 2 et n'incluent aucun critère pour gérer les comparaisons multiples avec un seul jeu de données. Or le risque de première espèce $(\alpha)$ pour $\mathrm{k}$ tests simultanés est de $1-\left(1-\alpha^{\mathrm{k}}\right)$ soit 0.4 au lieu de 0.05 pour les corrélations entre 5 variables [22]. Pour obtenir des tests d'hypothèses corrects, deux voies sont possibles (Legendre P., Université de Montréal, communication personnelle) : tester l'hypothèse générale englobant tous les tests simultanés puis effectuer chaque test sans correction ou bien corriger le niveau de chaque test. Nous avons testé l'hypothèse globale de la différence entre la moyenne multidimensionnelle des deux groupes (parcelles ou classes de taille) par un test de Mantel impliquant une matrice de similarité entre toutes les observations et une matrice modèle 
représentant la division des observations en deux groupes [23, 24]. Ce test équivaut à une analyse de variance multivariée non-paramétrique. Les similarités entre observations ont été calculées suivant le coefficient quantitatif et symétrique de Gower [24, équation 7.20 p 259]. Pour les corrélations, même paramétriques, aucun test global n'est disponible. Dans ce cas, nous avons appliqué la correction pour tests multiples dite de Bonferroni modifiée par Hochberg qui se déroule en deux étapes $[24,25]$. Tout d'abord il s'agit de tester l'hypothèse nulle sur l'ensemble des tests simultanés au niveau $\alpha$ en recherchant au moins un test significatif au niveau $\alpha /$ (nombre de tests simultanés). Ensuite pour tester l'hypothèse nulle pour chaque test, il faut ordonner les k probabilités en ordre croissant et les corriger par le facteur k-i + 1 (i étant le rang de la probabilité) puis les comparer à $\alpha$.

Par ailleurs, nous avons choisi de représenter les variables par leur médiane, leur quartile et leur étendue car ces grandeurs représentent beaucoup mieux la distribution dans le cas de variables ne suivant pas la loi normale. Cependant en cas de forte dissymétrie des distributions, la somme des médianes peut être légèrement supérieure à 100.

\section{Résultats}

Les contenus de gésiers préservés dans l'alcool se présentent sous la loupe comme une masse organo-minérale de texture fine (qui sera analysée au microscope) d'où émergent des minéraux et des fragments végétaux macroscopiques. Cette masse organo-minérale peut être clairement moulée dans le gésier disséqué ou constituer une série de petites boulettes. Seule leur forme distingue ces boulette qui, de ce fait, sont des agrégats galbés. Ces agrégats ont certainement été moulés par d'autres animaux et leur forte teneur en sol superficiel indique que ce sont des déjections de petits vers de terre. L'ensemble de la masse organo-minérale est piquetée de noir par de petits morceaux de charbon. Suivant la teneur en fragments végétaux et la manipulation, cette structure est plus au moins désagrégée.

Malgré une variabilité interindividuelle considérable (Fig. $1 \mathrm{a}$, b et c), nous constatons que les agrégats organo-minéraux des 13 individus (médiane $=77 \%$ ) sont majoritaires, les fragments végétaux $>50 \mu \mathrm{m}$ et les minéraux $>50 \mu \mathrm{m}$ sont minoritaires avec une médiane à respectivement $14 \% \mathrm{~V} / \mathrm{V}$ et $11 \% \mathrm{~V} / \mathrm{V}$. Ces agrégats organo-minéraux ont une teneur très élevée en fragments végétaux $<50 \mu \mathrm{m}(44 \% \mathrm{~V} / \mathrm{V})$ et leur teneur en matière organique totale $(51 \% \mathrm{~V} / \mathrm{V})$ est supérieure à tous les autres constituants micro et macroscopiques (Fig 1 b). Au total, la matière organique figurée représente $63 \%$ du volume des contenus de gésiers contre $6,5 \% \mathrm{~V} / \mathrm{V}$ pour la matière organique amorphe et $30 \% \mathrm{~V} / \mathrm{V}$ pour les minéraux (Fig $1 \mathrm{c}$ ).

Les individus des deux parcelles ont ingéré des déjections de vers de terre plus petits à l'exception d'un individu du pâturage amélioré. Ces agrégats appartiennent majoritairement à la classe 0.2-0.75 mm et composent de 9 à $71 \%$ de l'ensemble des contenus de gésiers (Fig. 1 a). Ces valeurs constituent une évaluation minimale des proportions en agrégats galbés car leur reconnaissance dépend du degré de conservation de la structure de l'échantillon, la dissection du gésier étant une source importante de dommages.

Les racines représentent $5 \%$ V/V pour les 13 individus étudiés, avec une médiane de $4 \%$ pour le pâturage amélioré et de $9 \%$ pour la savane naturelle (différence non significative). Les parties aériennes semblent moins abondantes (médiane globale à $0,2 \mathrm{~V} / \mathrm{V}, 2,5 \%$ pour la savane naturelle 
et $0 \%$ pour le pâturage amélioré, Fig. 2) mais les deux variables ne sont pas significativement différentes. Un individu du pâturage amélioré se distingue par la quantité de fragments végétaux macroscopiques $(47 \% \mathrm{~V} / \mathrm{V})$ à $70 \%$ constitués de parties aériennes. Cet individu est par ailleurs coprophage.

Des déjections holorganiques ne sont présentes en proportion importante que chez un unique individu du pâturage amélioré.

Peu de différences significatives ont été détectées entre les contenus des gésiers des individus de la savane naturelle et du pâturage amélioré. Les individus de la savane naturelle présentent plus de complexes organo-minéraux $(18,5 \%$ contre $6 \%$ ) et des teneurs en débris végétaux ( $8 \%$ contre $2 \%$ ) et de minéraux ( $6 \%$ contre $2 \%$ ) plus élevés dans ces complexes ainsi que moins de litière témoin et contre les galeries $\left(14 \mathrm{gm}^{-2}\right.$ et $97 \mathrm{gm}^{-2}$ pour la litière témoin, $37 \mathrm{gm}^{-2}$ et $78 \mathrm{gm}^{-2}$ pour la litière contre les galeries, pour savane naturelle et le pâturage amélioré respectivement) (Fig. 3).

La figure 4 présente les données relatives aux masses des individus, de litières et de matière organique légère du sol. Seule la quantité de litière témoin est corrélée significativement et négativement à une variable décrivant la teneur en matière organique des contenus de gésiers parmi les 16 variables analysées (Tab. 1) : le pourcentage volumique de fragments végétaux dans les complexes organo-minéraux $\left(\mathrm{r}^{2}=70,6 \%\right.$, Tab. 2$)$.

Les caractéristiques des gésiers mesurées à la loupe ne sont pas corrélées avec celles mesurées au microscope tandis que les quantités de litière témoin et galerie sont très fortement corrélées (Tab. 2).

L'abondance des particules organiques et minérales dans les contenus de gésiers forme une courbe bimodale en fonction de leur taille (Fig. 5 a et b) avec 2 granulométries privilégiées, la plus fine observée $(0,8 \mu \mathrm{m})$ et deux fractions grossières $(0,05 \mathrm{~mm}$ à $0,2 \mathrm{~mm})$. Une rupture sépare les fractions observées au microscope et celles observées à la loupe. Les deux échelles d'observation (loupe et microscope) correspondent probablement à une réalité alimentaire constituée pour partie de fèces de vers de terre (matière organique fine bien intégrée à la masse minérale) et pour partie de racines $>50 \mu \mathrm{m}$ (non-incorporée au sol) et de gros grains minéraux.

\section{Discussion}

\subsection{Un régime alimentaire d'endogé polyhumique ou un régime anécique à redéfinir}

En convertissant les pourcentages volumiques en pourcentages massiques avec pour densités particulaires ou réelles $2.7,2.2$ et $1 \mathrm{~g} . \mathrm{cm}^{-3}$, respectivement pour la matière minérale, matière organique amorphe et matière organique figurée (estimations [26, 27]), les contenus de gésiers analysés ont 38,5 \% de matière organique figurée (avec 8,5 \% de fragments macroscopiques dont une majorité de racines), $7 \%$ de matière organique amorphe et $54 \%$ de matière minérale. Cela pourrait tout à fait correspondre à un régime alimentaire d'anécique si $77 \%$ des débris végétaux n'étaient pas microscopiques et déjà incorporés à des agrégats organo-minéraux. L'identification de la majorité de ces agrégats comme des déjections de vers de terre implique que ce matériel est incorporé au sol par d'autres vers de terre. Dès lors, la litière de surface directement ingérée par

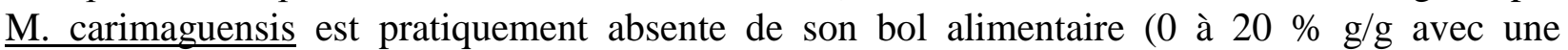
médiane à 0 ). Seul un des individus analysé incorpore une proportion important de litière de 
surface et a donc un régime alimentaire d'anécique. Les traits dominants du régime alimentaire de

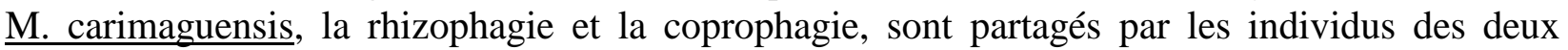
milieux analysés bien que la qualité de l'incorporation de la matière organique à la matière minérale soit meilleure pour les individus de savane naturelle, à ce stade de la digestion.

Les proportions élevées de sol et de fragments végétaux dans les fèces ingérées par $\underline{M}$. carimaguensis indiquent qu'elles ont été produites par des vers de terre polyhumiques. D'après leurs masses moyennes à l'état adulte et leurs diamètres périclitellaires [14], les 3 espèces de vers de terre endogés présentes sur les sites, Andiodrilus sp., Glossodrilus sp. et Ocnerodrilidae sp.,

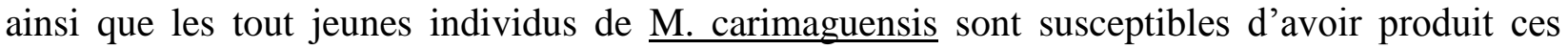
fèces. Réciproquement, la plus petite de ces espèces, Ocnerodrilidae sp. a été observé fréquemment se nourrissant des fèces souterraines de $\underline{M}$. carimaguensis [28]. Cette coprophagie placerait $\underline{M}$. carimaguensis à la fin de la chaîne trophique du sol tandis que l'incorporation de la litière au sol, fonction associée aux anéciques, serait typique du début de cette chaîne. En fait la consommation de quantités importantes de déjections de vers de terre associée à de la litière montre la complexité des réseaux trophiques dans le sol où des niveaux trophiques bien tranchés sont rarement observés [29].

L'exploration du sol par M. carimaguensis pour sélectionner des fèces est très improbable du fait de la forme de ses galeries, certainement en $U$, et de ses sorties assez fréquentes à la surface (Jiménez, Decaëns et Mariani données non publiées). De plus les fèces des endogés sont relativement fréquentes à la surface du sol comme l'atteste une cartographie détaillée des biostructures superficielles de la savane naturelle étudiée ici (Mariani, Decaëns et Jiménez données non publiées). Cette étude révèle en effet que les fèces superficielles de Glossodrilus sp. et Ocnerodrilidae sp. sont absents ou très rares tandis que ceux de Andiodrilus sp. sont au contraire assez abondants puisqu'ils couvrent 2,2\% de la surface du sol avec toutefois une forte hétérogénéité spatiale (coefficient de variation 132\%). Toutefois l'étude de l'humus et de la litière, en particulier de la distribution verticale des fèces, est indispensable pour cerner les déterminants de l'ingestion de la masse organo-minérale par $\underline{\mathrm{M}}$. carimaguensis et leur attribuer une valeur écologique. Si l'hypothèse d'une ingestion sélective de la masse organo-minérale à la surface du sol est confortée, alors ce régime particulier est le fait d'un comportement anécique dont le sens écologique se trouve modifié. Il pourrait être dû à une adaptation à l'absence de litière dans les savanes régulièrement brûlées.

\subsection{La variabilité temporelle de l'alimentation de $\underline{\text { M. carimaguensis }}$}

L'analyse des turricules superficiels fournit des résultats incohérents avec notre étude et atteste l'incorporation de litière fraîche à la masse organo-minérale par $\underline{M}$. carimaguensis [30, 31]. Ces fragments de litière pourraient toutefois en partie avoir été inclus au turricule au moment de son excrétion. Ces turricules présenteraient de fait deux types de fragments végétaux, les premiers, trop grands et trop rigides pour avoir été ingérés par les vers, ont probablement été recouverts ; les seconds correspondent à de très fins fragments (quelques $\mathrm{mm}$ ), visibles après la destruction du cortex lisse lors de simulations de pluie (Mariani et collaborateurs, données non publiées) et qui pourraient avoir subi le transit intestinal. Les turricules récoltés dans les mêmes parcelles et à la même époque que l'étude que nous présentons ici se sont révélés plus riches en graines que le sol témoin (Decaëns et collaborateurs, données non publiées). $\mathrm{M}$. carimaguensis opérerait donc une sélection que notre étude des contenus de gésiers n'a pas retrouvée. Des lames minces de 
turricules frais de $\underline{\mathrm{M}}$. carimaguensis, collectées dans le pâturage amélioré et la savane naturelle de notre étude, révèlent une grande hétérogénéité dans la distribution des fragments végétaux fins [15]. Cette hétérogénéité pourrait être expliquée par une variabilité du régime alimentaire de $\underline{M}$. carimaguensis à l'échelle de temps de formation d'un turricule qui est de 3 jours en moyenne et de 3 semaines au maximum ([15], Decaëns et Jiménez comm. pers.). Selon cette hypothèse, $\underline{M}$. carimaguensis pourrait adopter periodiquement un régime anécique typique. Cette variabilité dans l'utilisation des ressources pourrait en outre expliquer les variations inter-individuelles fortes du régime alimentaire constatées dans cette étude.

\section{Conclusion}

Cette étude montre que l'alimentation de $\underline{M}$. carimaguensis ne correspond pas à un simple mélange de sol et de litière et qu'il peut exploiter les racines et les masses de déjections de vers de terre (notamment celles qui sont de petit diamètre). Des analyses détaillées de l'humus et de la litière ainsi que de contenus digestifs au cours de la période nycthémérale sont nécessaires pour découvrir de quelle façon et par quel acteur se fait l'incorporation de la litière au sol et pour

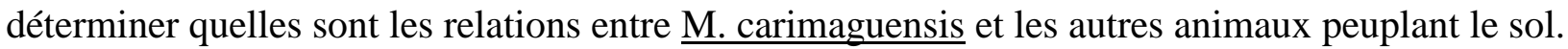

\section{Remerciements}

Nous sommes reconnaissants envers P. Lavelle du LEST (IRD/ Université de Paris VI, France) pour de précieux commentaires sur le manuscrit de cet article, envers le personnel de la station de recherche Carimagua, Colombie, pour la réalisation de l'échantillonnage et envers P. Margerie de l'université de Rouen, France, pour son aide concernant l'identification des fragments végétaux.

\section{Bibliographie}

[1] Lavelle P., Brussaard L., Hendrix P., Earthworm management in tropical Agrosystems, CABI publishing, Oxon and New York, 1999.

[2] Senapati B.K., Lavelle P., Giri S., Pashanasi B., Alegre J., Decaëns T., Jimenez J.J., Albrecht A., Blanchart E., Mahieux M., Rousseaux L., Thomas R., Panigrahi P.K., Venkatachalam M., Insoil earthworm technologies for tropical ecosystems, in : Lavelle P., Brussaard L., Hendrix P. (Eds), Earthworm management in tropical Agrosystems, CABI publishing, Oxon and New York, 1999, pp. 199-238.

[3] Bouché M.B., Stratégies lombriciennes, in : Lohm U., Persson T. (Eds), Soil Organism as Components of Ecosystems, Ecology Bulletin (Stockolm), 1977, pp. 122-132.

[4] Piearce T.G., The calcium relations of selected Lumbricidæ, J. Anim. Ecol. 41 (1972) 167188.

[5] Bouché M.B., Kretzschmar A., Fonction des lombriciens. II. Recherches méthodologiques pour l'analyse du sol ingéré (étude du peuplement de la station R.C.P.-165/P.B.I.), Rev. Ecol. Biol. Sol 11 (1974) 127-139.

[6] Bolton P.J., Phillipson J., Burrowing, feeding, egestion and energy budget of Allolobophora rosea (Savigny) (Lumbricidæ), Oecologia (Berlin) 23 (1976) 225-245.

[7] Ferrière G., Fonctions des lombriciens VII. Une méthode d'anlyse de la matière organique végétale ingérée, Pedobiologia 20 (1980) 263-273. 
[8] Kanyonyo Kakajondo J.B., Ecologie alimentaire du ver de terre detritivore Millsonia lamtoiana (Acanthdrilidae, Oligochètes) dans la savane de Lamto (Côte d'Ivoire), thèse de doctorat, Paris VI, 1984.

[9] James S.W., Seastedt T.R., Nitogen mineralization by native and introduced earthworms: effects on big bluestem growth, Ecology 67 (1986) 1094-1097.

[10] James S.W., Cunningham M.R., Feeding ecology of some earthworms in Kansas tallgrass prairie, Am. Midl. Nat. 121 (1989) 78-83.

[11] Judas M., Gut content analysis of earthworms (Lumbricidae) in a beechwood, Soil Biol. Biochem. 24 (1992) 1413-1417.

[12] Bernier N., Earthworm feeding activity and development of the humus profile, Biol. Fertil. Soils 26 (1998) 215-223.

[13] Jiménez J.J., Moreno A.G., Decaëns T., Lavelle P., Fisher M., Thomas R.J., Earthworm communities in natives savannas and man-made pastures of the Eastern Plains of Colombia., Biol. Fertil. Soils 28 (1998) 101-110.

[14] Decaëns T., Lavelle P., Jimenez Jaen J.J., Escobar G., Rippstein G., Impact of land management on soil macrofauna in the Oriental Llanos of Colombia, Eur. J. Soil Biol. 30 (1994) 157-168.

[15] Decaens T., Degradation dynamics of surface earthworm casts in grasslands of the eastern plains of Colombia, Biol. Fertil. Soils 32 (2000) 149-156.

[16] Decaëns T., Rangel A.F., Asakawa N., Thomas R., Carbon and nitrogen dynamics in in-situ ageing earthworm casts in grasslands of the eastern Plains of Colombia, Biol. Fertil. Soils 30 (1999) 20-28.

[17] Decaëns T., Mariani L., Lavelle P., Soil surface macrofaunal communities associated with earthworm casts in grasslands of the Eastern Plains of Colombia., Appl. Soil Ecol. 13 (1999) 87100.

[18] Decaëns T., Jimenez J.J., Lavelle P., Effect of exclusion of the anecic earthworm Martiodrilus carimagunesis Jimenez and Moreno on soil properties and plant growth in grasslands of the eastern plains of Colombia., Pedobiologia 43 (1999) 1-7.

[19] Fisher M.J., Rao I.M., Ayarza M.A., Lascano C.E., Sanz J.I., Thomas R.J., Vera R.R., Carbon storage by introduced deep-rooted grasses in the South American savannas, Nature 371 (1994) 236-238.

[20] Sokal R.R., Rohlf F.J., Biometry: the principles and practice of statistics in biological research. 3d ed. 1995, New York: W.H. Freeman and company.

[21] Legendre P., A.Vaudor., The $\mathrm{R}$ package: multidimentional analysis, spatial analysis. Université de Montréal, Département des Sciences Biologiques, Montréal, 1991.

[22] Scherrer B., Biostatistique. 1984, Montréal Paris Casablanca: Gaëtan Morin éditeur Ltée.

[23] Legendre P., Fortin M.J., Spatial pattern and ecological analysis, Vegetatio 80 (1989) 107138. 
[24] Legendre L., Legendre P., Numerical Ecology. Second english edition. Developments in environmental modelling 20. 1998, Amsterdam: Elsevier.

[25] Hochberg Y., A sharper Bonferroni procedure for multiple tests of significance, Biometrika 75 (1988) 800-802.

[26] Christensen B.T., Physical Fractionantion of Soil and Organic Matter in Primary Particle Size and Density Separates, Adv Soil Sci 20 (1992) 1-90.

[27] Bernier N., Altitudinal changes in humus form dynamics in a spruce forest at the montane level, Plant and Soil 178 (1996) 1-28.

[28] Jiménez J.J., Moreno A., Lavelle P., Decaëns T., Population dynamics and adaptative strategies of Martiodrilus carimaguensis (Oligochaeta, Glossoscolacidae), a native species from the well-drained savannas of Colombia, Appl. Soil Ecol. 9 (1998) 153 -160.

[29] Ponsard S., Arditi R., What can stable isotopes (d15N and d13C) tell about the food web of soil macroinvertebrates?, Ecology 81 (2000) 852-864.

[30] Guggenberger G., Zech W., Thomas R.J., Lignin and carbohydrate alteration in particle-size separates of an oxisol under tropical pastures following native savanna, Soil Biol. Biochem. 27 (1995) 1629-1638.

[31] Guggenberger G., Thomas R.J., Zech W., Soil organic matter within earthworm casts of an anecic-endogeic tropical pasture community, Colombia, Appl. Soil Ecol. 3 (1996) 263-274. 


\section{Figures}

Figure 1. Les caractéristiques des contenus de gésiers communes aux 2 parcelles.

a) Eléments macroscopiques ( $>50 \mu \mathrm{m})$

b)Eléments microscopiques $(<50 \mu \mathrm{m})$

c)Total

M.O. : matière organique. Autres agrégats : anguleux et indéterminés.

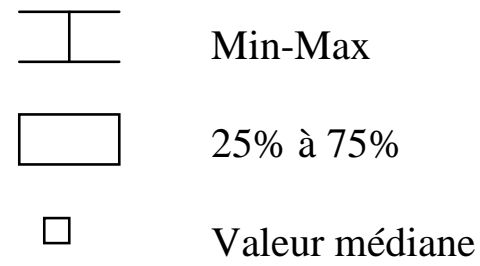

Figure 2. Nature des fragments végétaux macroscopiques pour l'ensemble des contenus de gésiers.

Lecture du graphique : voir figure 1.

Figure 3. Comparaison entre le pâturage amélioré et la savane naturelle.

Variables statistiquement différantes pour les 2 parcelles parmi les 16 du tableau 1.

a) Complexes organo-minéraux totaux

b) Matière organique figurée dans les complexes organo-minéraux

c) Matière organique amorphe dans les complexes organo-minéraux

d) Litière témoin

e) Litière galerie.

Lecture du graphique : voir figure 1.

Figure 4. Masse des individus et caractéristiques des parcelles

a) Masse de la litière de litière (en $\mathrm{g} / \mathrm{m}^{2}$ ).

b) Masse des individus (en grammes) et de la matière organique figurée du sol (en $\mathrm{mg} / \mathrm{g}$ de sol). Lecture du graphique : voir figure 1.

Figure 5. Densité de probabilité des particules organiques et minérales des contenus de gésiers (échelle logarithmique).

a. Particules organiques

b. Particules minérales 


\section{LEGENDES}

Tableau 1. Les 16 variables impliquées dans les corrélations et la comparaison des parcelles.

Tableau 2. Corrélations significatives entre les variables détaillées au tableau 1.

$*$ : test significatif à $5 \% * * *$ : test significatif à $0.5 \%$. 

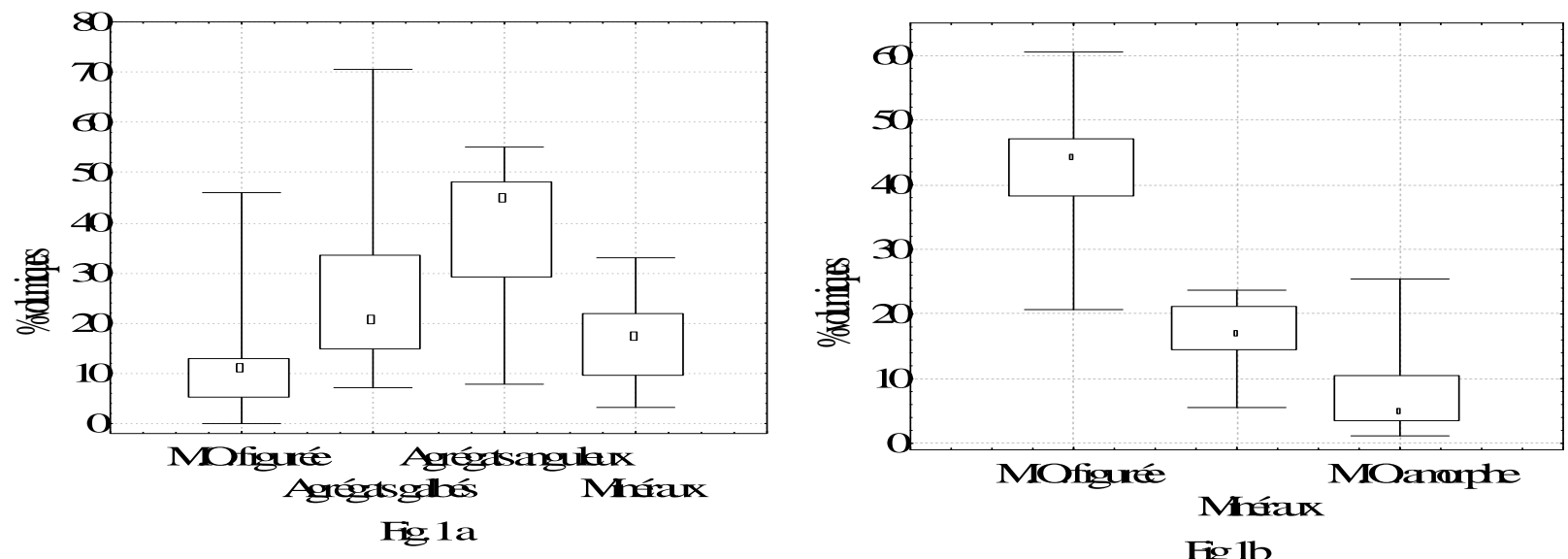

Fig $1 b$

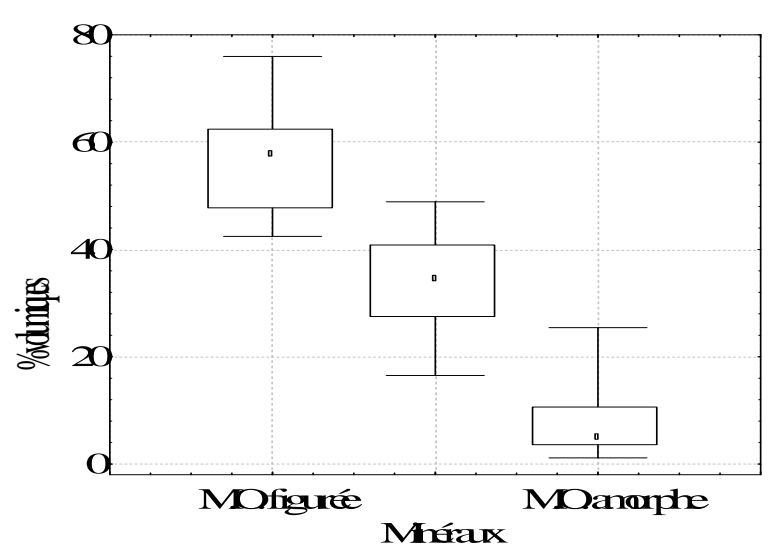

Fig 1c
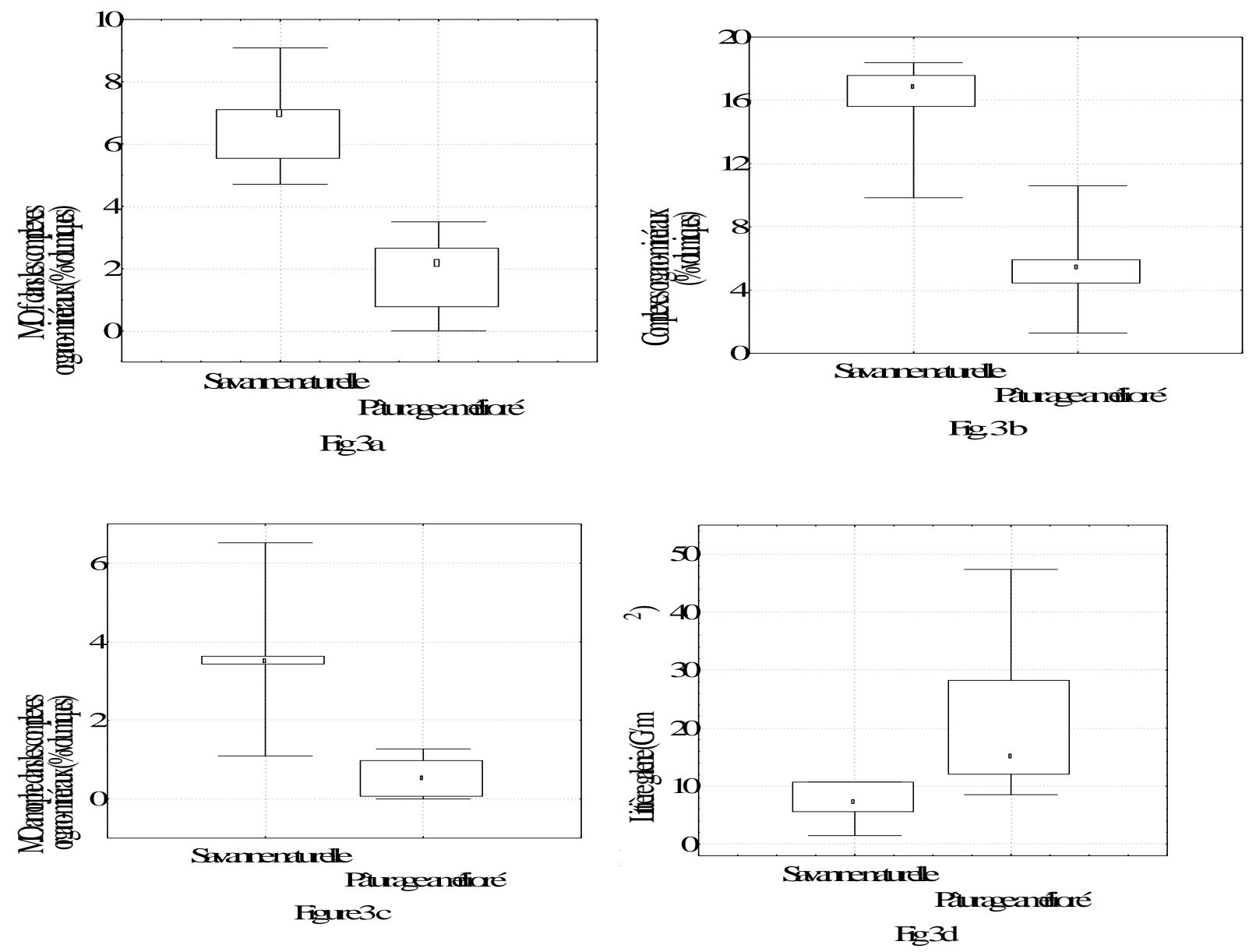

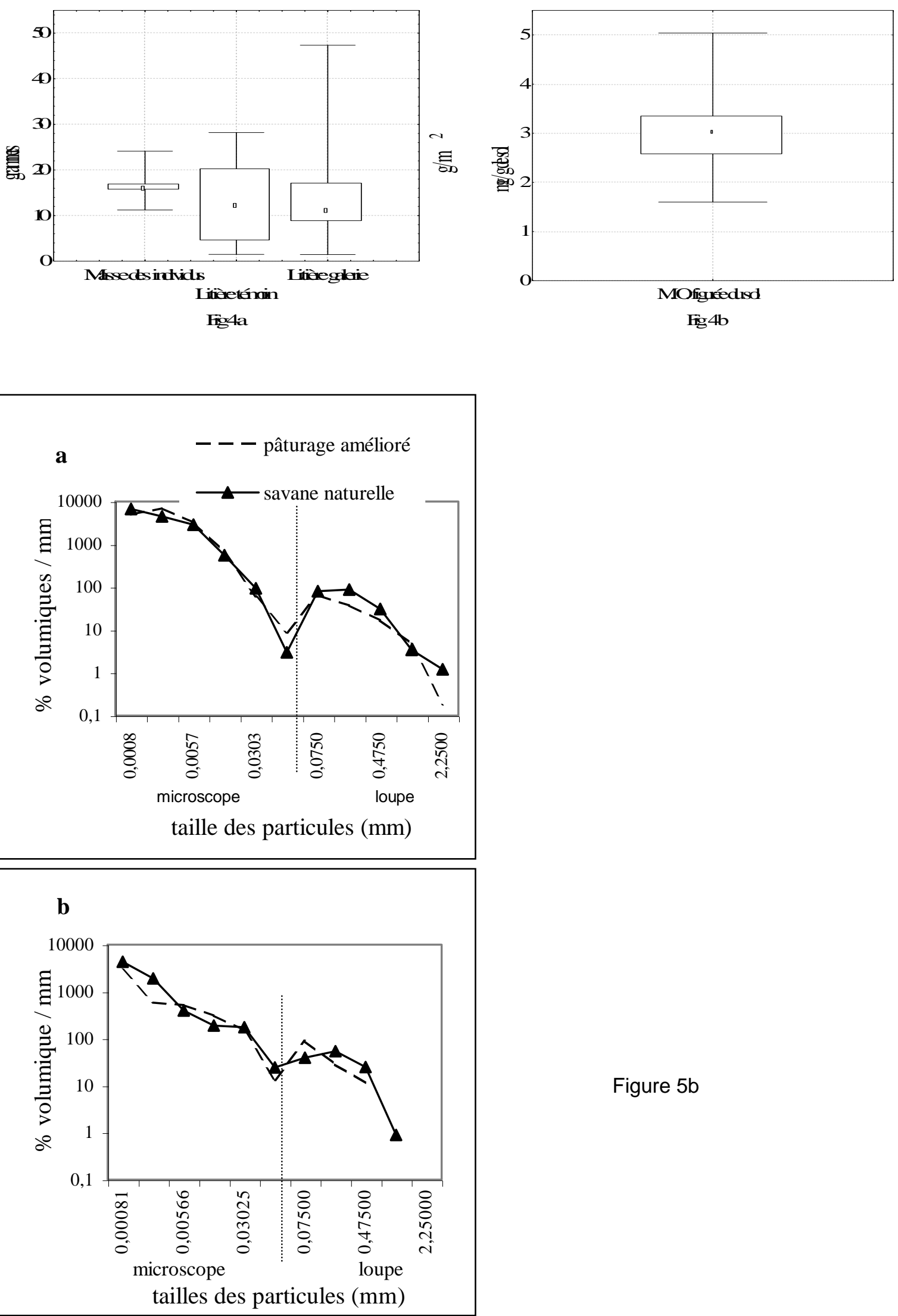

Figure $5 b$ 


\begin{tabular}{|c|c|}
\hline \multirow{3}{*}{$\begin{array}{c}\text { Observations à la loupe en } \% \\
\text { volumiques }\end{array}$} & Fragments végétaux \\
\hline & Agrégats \\
\hline & Minéraux \\
\hline \multirow{9}{*}{$\begin{array}{l}\text { Observations au microscope } \\
\text { en } \% \text { volumiques }\end{array}$} & $\begin{array}{c}\text { Fragments végétaux et } \\
\text { bactéries totaux }\end{array}$ \\
\hline & Minéraux totaux \\
\hline & $\begin{array}{c}\text { Fragments végétaux et } \\
\text { bactéries dans les complexes } \\
\text { organo-minéraux }\end{array}$ \\
\hline & $\begin{array}{c}\text { Minéraux dans les complexes } \\
\text { organo-minéraux }\end{array}$ \\
\hline & $\begin{array}{c}\text { M. O. amorphe dans les } \\
\text { complexes organo-minéraux }\end{array}$ \\
\hline & $\begin{array}{c}\text { Complexes organo-minéraux } \\
\text { totaux }\end{array}$ \\
\hline & M. O. amorphe libre \\
\hline & M. O. amorphe totale \\
\hline & M. O. totale \\
\hline grammes & $\begin{array}{c}\text { masse des individus dans } \\
\text { l'alcool }\end{array}$ \\
\hline $\mathrm{mg} / \mathrm{g}$ de sol & Fragments végétaux du sol \\
\hline \multirow[t]{2}{*}{$\mathrm{g} / \mathrm{m}^{2}$} & litière témoin \\
\hline & litière galerie \\
\hline
\end{tabular}

Tableau 1

Littière témoin

\begin{tabular}{ll}
\hline Littière galerie & $0.90 * * *$ \\
\hline $\begin{array}{l}\text { Fragments végétaux } \\
\text { dans les complexes } \\
\text { organo-minéraux }\end{array}$ & $-0.84 *$ \\
\hline
\end{tabular}

Tableau 2 